\title{
On soft filters of soft BE-algebras
}

\author{
Yilmaz Ceven and Zekiye Ciloglu Sahin \\ Department of Mathematics, Faculty of Arts and Sciences, Suleyman Demirel University, Isparta, Turkey
}

Received: 8 February 2017, Accepted: 8 SEptember 2017

Published online: 17 February 2018.

\begin{abstract}
In this paper, we apply the notion of soft sets by Molodtsov to the theory of BE-algebras. For this purpose, we introduced the notions of dual filter, S-filter and dual S-filter in BE-algebra and also introduced the notions of soft filter, soft F-filter, soft dual filter and soft dual F-filter in a soft BE-algebra. Then we derive their basic properties.
\end{abstract}

Keywords: BE-algebra, filter, dual filter, soft filter, soft dual filter.

\section{Introduction}

Imai and Iséki [8 ] and Iséki [9 ] introduced BCK-algebras and BCI-algebras in 1966. As a generalization of a BCK-algebra, H. S. Kim and Y. H. Kim [14] introduced the notion of a BE-algebra and investigated some properties. Ahn and So introduced the notion of ideals in BE-algebras and gave some properties of ideals in BE-algebras in [2].

Many problems in system identification involve characteristics which are essentially nonprobabilistic in nature [19]. Zadeh [20] introduced fuzzy set theory as an alternative to probability theory. The approach to uncertainty is outlined by Zadeh in [21]. Uncertainties can not be handled using traditional mathematical tools but may be dealt with some theories such as probability theory, theories of (intuitionistic) fuzzy sets, vague sets, interval mathematics and rough sets. These theories have some difficulties which are pointed out in [17]. In [16] and [17], Maji et al. and Molodtsov suggested that one reason for these difficulties may be due to the inadequacy of the parametrization tool of the theory. To overcome those difficulties, Molodtsov [17] introduced the concept of soft set as a new mathematical tool for dealing with uncertainties and pointed out several directions for the applications of soft sets. Maji et al. [15,16] described the application of soft set theory to a decision-making problem and studied several operations on the theory of soft sets. In [4], Chen et al. presented a new definition of soft set parametrization reduction and compared this definition to the related concept of attributes reduction in rough set theory. Çağman et al. introduced fuzzy parameterized (FP) soft sets and their related properties in [5]. Feng [7] worked the application of soft rough approximations in multicriteria group decision-making problems. In [3], Aktaş and Çağman studied the basic concepts of soft set theory, compared soft sets to fuzzy and rough sets providing examples to clarify their differences and discussed the notion of soft groups. Jun [10] introduced the notion of soft BCK/BCI-algebras and derived their basic properties. In [12], Jun and Park introduced the notion of soft ideals and idealistic soft BCK/BCI-algebras and investigated some related properties. In [18], Park et al. introduced the notion of soft ws-algebras, soft subalgebras and soft deductive systems and derived their basic properties. In $[1,11,13]$, researchers studied on applications of soft sets in BE-algebras.

In this paper, we apply the notion of soft sets by Molodtsov to the theory of BE-algebras. For this purpose, we introduced the notions of dual filter, S-filter and dual S-filter in BE-algebra and also introduced the notions of soft filter, soft F-filter, soft dual filter and soft dual F-filter in a soft BE-algebra. Then we derive their basic properties.

*Corresponding author e-mail: yilmazceven@sdu.edu.tr

(C) 2018 BISKA Bilisim Technology 


\section{Preliminaries}

We recall some definitions and results.

Definition 1. An algebra $(X ; *, 1)$ of type $(2,0)$ is called a BE-algebra ([14]) if

(BE1) $x * x=1$,

(BE2) $x * 1=1$,

(BE3) $1 * x=x$,

(BE4) $x *(y * z)=y *(x * z)$, for all $x, y, z \in X$.

A relation " $\leq$ " on $X$ is defined by

$$
x \leq y \Longleftrightarrow x * y=1 \text {. }
$$

for all $x, y \in X$.

A non-empty subset $A$ of a BE-algebra $X$ is said to be a subalgebra of $X$ if $x * y \in A$ for all $x, y \in A$.

Let $X$ and $Y$ be two BE-algebra. If the mapping $f: X \longrightarrow Y$ satisfies the condition $f(x * y)=f(x) * f(y)$ for all $x, y \in X$, $f$ is called a BE-algebra homomorphism.

Definition 2. [6] Let $X$ be a BE-algebra. If there exists an element 0 satisfying $0 \leq x($ or $0 * x=1)$ for all $x \in X$, then the element 0 is called unit of X. A BE-algebra with unit is called a bounded BE-algebra.

In a bounded BE-algebra, $x * 0$ is denoted by $x N$.

Definition 3. Let $(X ; *, 1)$ be a BE-algebra and let $A$ be a non-empty subset of X. Then A is called a filter of X (see [14]) if

(A1) $1 \in A$;

(A2) $x \in A, x * y \in A \Rightarrow y \in A$, for all $x, y \in X$.

A soft set theory is introduced by Molodtsov [17].

Definition 4. Let $U$ be an initial universe set and $E$ be a set of parameters. Let $P(U)$ denote the power set of $U$ and $A$ be a subset of $E$. A pair $(F, A)$ is called a soft set over $U$, where $F$ is a mapping given by $F: A \longrightarrow P(U)$.

Let $(F, A)$ and $(G, B)$ be two soft sets over a common universe $U$. The intersection of the soft sets $(F, A)$ and $(G, B)$ is defined to be the soft set $(H, C)$ satisfying the following conditions:

(i) $C=A \cap B$,

(ii) $H(x)=F(x)$ or $G(x)$ for all $x \in C$, (as both are same sets ).

In this case, we write $(F, A) \widetilde{\cap}(G, B)=(H, C)$. The union of the soft sets $(F, A)$ and $(G, B)$ is defined to be the soft set $(H, C)$ satisfying the following conditions:

(i) $C=A \cup B$.

(ii) $H(x)= \begin{cases}F(x), & \text { if } x \in A \backslash B, \\ G(x), & \text { if } x \in B \backslash A, \text { for all } x \in C . \\ F(x) \cup G(x), & \text { if } x \in A \cap B\end{cases}$

In this case, we write $(F, A) \tilde{\cup}(G, B)=(H, C)$. " $(F, A) \operatorname{AND}(G, B)$ " is denoted by $(F, A) \sim(G, B)$ and defined by

$$
(F, A) \tilde{\wedge}(G, B)=(H, A \times B)
$$


where $H(a, b)=F(a) \cap G(b)$ for all $(a, b) \in A \times B$ and $A \times B$ is the Cartesian product of the sets $A$ and $B$." $(F, A)$ OR $(G, B)$ " is denoted by $(F, A) \widetilde{\vee}(G, B)$ and defined by

$$
(F, A) \tilde{\vee}(G, B)=(H, A \times B),
$$

where $H(a, b)=F(a) \cup G(b)$ for all $(a, b) \in A \times B$.

\section{Main part}

In what follows, we take $X$ as a BE-algebra and a set of parameters unless otherwise specified.

Definition 5. Let $S$ be a subalgebra of $X$ and $A$ be a subset of $X$. Then $A$ is called a S-filter of $X$ if

(S1) $1 \in A$;

(S2) $x \in A, x * y \in A \Rightarrow y \in A$, for all $y \in S$ and $x \in X$.

If $A$ is a subset of $X$ contains $S$, then $A$ is a $S$-filter of $X$. Every filter of $X$ is a $S$-filter of $X$. But, generally, every $S$-filter of $X$ is not a filter of $X$.

Example 1. Consider the set $X=\{1, a, b, c, d\}$ with the following table:

\begin{tabular}{c|ccccc}
$*$ & 1 & $a$ & $b$ & $c$ & $d$ \\
\hline 1 & 1 & $a$ & $b$ & $c$ & $d$ \\
$a$ & 1 & 1 & $b$ & $c$ & $d$ \\
$b$ & 1 & $a$ & 1 & $c$ & $c$ \\
$c$ & 1 & 1 & $b$ & 1 & $b$ \\
$d$ & 1 & 1 & 1 & 1 & 1
\end{tabular}.

Then $X$ is a BE-algebra [11]. The set $A=\{1, b, c\}$ is not a filter of $X$ because $b \in A, b * d=c \in A$ but $d \notin A$. The set $A$ is a $S$-filter where $S=\{1, b\}$.

Definition 6. Let $X$ be a bounded BE-algebra with unit $O$ and $D$ be a nonempty subset of $X$.Then $D$ is called a dual filter of $X$ if

(D1) $0 \in D$,

(D2) $x \in D,(x N * y N) N \in D \Longrightarrow y \in D$, for any $x, y \in X$.

Definition 7. Let $X$ be a bounded BE-algebra with unit $0, S$ be a subalgebra of $X$ and $D$ be a nonempty subset of $X$.Then $D$ is called a dual $S$-filter of $X$ if

(DS1) $0 \in D$,

(DS2) $x \in D,(x N * y N) N \in D \Longrightarrow y \in D$, for any $y \in S$ and $x \in X$.

If $D$ is a subset of $X$ contains $S$, then $D$ is a dual $S$-filter of $X$. Every dual filter of $X$ is a dual $S$-filter of $X$. But, generally, every dual $S$-filter of $X$ is not a dual filter of $X$.

Example 2. In Example 1, $X$ is a bounded BE-algebra with unit element $d$. The set $D=\{1, d\}$ is not a dual filter of $X$ because $d \in D,(d N * a N) N=((d * d) *(a * d)) * d=(1 * d) * d=d * d=1 \in D$ but $a \notin D$. The set $D$ is a dual $S$-filter of $X$ where $S=\{1, d\}$. The set $D_{1}=\{b, d\}$ is a dual filter of $X$.

Definition 8. Let $E$ be a set of parameters and A a subset of $E$. A pair $(F, A)$ is called a soft set over $X$ where $F$ is a mapping given by $F: A \longrightarrow P(X)$. 
Definition 9. Let $(F, A)$ be a soft set over $X .(F, A)$ is called a soft BE-algebra over $X$ if $F(x)$ is a subalgebra of $X$ for all $x \in A$.

Example 3. Consider the BE-algebra in Example 3.1. Then $(F, X)$ is a soft BE-algebra over $X$ with the following mapping:

$$
F: X \longrightarrow P(X), F(1)=\{1\}, F(a)=\{1, a\}, F(b)=\{1, b\}, F(c)=\{1, c\}, F(d)=\{1, d\}
$$

Definition 10. Let $(F, A)$ be a soft set over $X .(F, A)$ is called a soft filter over $X$ if $F(x)$ is a filter of $X$ for all $x \in A$.

Example 4. Consider the BE-algebra in Example 3.1 and the mapping defined by $F: X \longrightarrow P(X), F(1)=\{1\}, F(a)=$ $\{1, a\}, F(b)=\{1, b\}, F(c)=\{1, a, c\}, F(d)=X$. Since $\{1\},\{1, a\},\{1, b\},\{1, a, c\}$ and $X$ are filters of $X$, the soft set $(F, X)$ is a soft filter over $X$.

Definition 11. Let $(F, A)$ be a soft BE-algebra and $(G, B)$ be a soft set over $X .(G, B)$ is called a soft $F$-filter over $X$ if

(i) $B \subseteq A$,

(ii) $G(x)$ is a $F(x)$-filter of $X$ for all $x \in B$.

Example 5. Consider the set $X=\{1, a, b, c\}$ with the following table:

\begin{tabular}{c|cccc}
$*$ & 1 & $a$ & $b$ & $c$ \\
\hline 1 & 1 & $a$ & $b$ & $c$ \\
$a$ & 1 & 1 & $a$ & $a$ \\
$b$ & 1 & 1 & 1 & $a$ \\
$c$ & 1 & $a$ & $a$ & 1
\end{tabular}

Then $X$ is a BE-algebra [13]. The soft set $(F, X)$ defined by $F: X \longrightarrow P(X), F(1)=\{1\}, F(a)=\{1, a\}, F(b)=\{1, b\}$, $F(c)=\{1, a, c\}$ is a soft BE-algebra. Consider the set $B=\{1, a, b\}$ and the mapping defined by $G: B \longrightarrow P(X), G(1)=$ $\{1\}, G(a)=\{1, a, b\}, G(b)=\{1, a, c\}$. Then $(G, B)$ is a soft set over $X$. It is easily seen that $B \subseteq X$ and $G(1)$ is a $F(1)$-filter, $G(a)$ is a $F(a)$-filter, $G(b)$ is a $F(b)$-filter. Hence $(G, B)$ is a soft $F$-filter over $X$.

If $F(x)$ is a subset of $G(x)$ for all $x \in B$, then $(G, B)$ is a soft $F$-filter over $X$. Every soft filter is a soft $F$-filter over $X$. But, generally, every soft $F$-filter is not a soft filter over $X$.

Example 6. In Example 5, $(G, B)$ is a soft $F$-filter but is not a soft filter over $X$ because $G(a)$ is not a filter of $X$ since $a \in B, a * c=a \in B$ but $c \notin B$.

Definition 12. Let $X$ be a bounded BE-algebra and $(F, A)$ be a soft set over $X$. Then $(F, A)$ is called a soft dual filter over $X$ if $F(x)$ is a dual filter of $X$ for all $x \in A$.

Definition 13. Let $X$ be a bounded BE-algebra, $(F, A)$ be a soft BE-algebra and $(G, B)$ be a soft set over $X$. Then $(G, B)$ is called a soft dual $F$-filter over $X$ if

(i) $B \subseteq A$,

(ii) $G(x)$ is a soft dual $F(x)$-filter over $X$ for all $x \in B$.

Example 7. Consider the BE-algebra in Example 1. Then $(F, X)$ is a soft set over $X$ with the following mapping:

$$
F: X \longrightarrow P(X), F(1)=F(a)=X, F(b)=\{b, d\}, F(c)=\{d, c\}, F(d)=\{d\}
$$

We see that $F(x)$ is a dual filter over $X$ for all $x \in X$. Hence, $(F, A)$ is a soft dual filter over $X$. 
Example 8. Consider the BE-algebra in Example 1. Then $(F, X)$ is a soft BE-algebra over $X$ with the following mapping:

$$
F: X \longrightarrow P(X), F(1)=\{1\}, F(a)=\{1, a\}, F(b)=\{1, b\}, F(c)=\{1, c\}, F(d)=\{1, d\} .
$$

Now let $B=\{1, a, d\}$ and consider the mapping $G: B \longrightarrow P(X)$ where $G(1)=G(d)=\{1, d\}, G(a)=\{d\}$. Then $G(1)$ is a soft dual $F(1)$-filter, $G(a)$ is a soft dual $F(a)$-filter and $G(d)$ is a soft dual $F(d)$-filter over $X$. Therefore $(G, B)$ is a soft dual $F$-filter over $X$.

If $F(x) \subseteq B(x)$ for all $x \in B,(G, B)$ is a soft dual $F$-filter over $X$. Every soft dual filter is a soft dual $F$-filter over $X$. But, generally, every soft dual $F$-filter is not a soft dual filter over $X$.

Example 9. In Example 8, $(G, B)$ is a soft dual $F$-filter but not a soft dual filter over $X$. Because $G(1)=\{1, d\}$ is not a dual filter of $X$ since $d \in G(1),(d N * a N) N=1 \in G(1)$ but $a \notin G(1)$.

Theorem 1. (i) Let $(F, A)$ be a soft BE-algebra (soft filter) over X. If $C$ is a subset of $A,\left(\left.F\right|_{C}, C\right)$ is a soft BE-algebra (soft filter) over $X$.

(ii) Let $(F, A)$ be a soft BE-algebra and $(G, B)$ be a soft $F$-filter over $X$. If $C$ is a subset of $B,\left(\left.G\right|_{C}, C\right)$ is a soft $F$-filter over $X$.

(iii) Let $X$ be a bounded BE-algebra and $(F, A)$ be a soft dual filter. If $C$ is a subset of $A,\left(F \mid{ }_{C}, C\right)$ is a soft dual filter over $X$.

(iv) Let $X$ be a bounded BE-algebra, $(F, A)$ be a soft BE-algebra and $(G, B)$ be a soft dual F-filter over $X$. If $C$ is a subset of $B,\left(\left.G\right|_{C}, C\right)$ is a soft dual $F$-filter over $X$.

Proof. Straightforward.

Although $(F, A)$ is not a soft filter over $X$, there can be a subset $C$ of $A$ such that $\left(\left.F\right|_{C}, C\right)$ is a soft filter over $X$. Furthermore, although $(F, A)$ is not a soft dual filter over $X$, there can be a subset $C$ of $A$ such that $\left(\left.F\right|_{C}, C\right)$ is a soft dual filter over $X$.

Example 10. (1) Consider the BE-algebra in Example 1. Let $(F, X)$ be a soft set over $X$ with the following mapping: $F: X \longrightarrow P(X), F(1)=\{1\}, F(a)=\{1, a\}, F(b)=\{1, b, c\}, F(c)=\{1, a, c\}, F(d)=X$. Then $(F, A)$ is not a soft filter. For the subset $C=\{1, a, c\}$ of $A$, the soft set $\left(\left.F\right|_{C}, C\right)$ is a soft filter.

(2) Consider the BE-algebra in Example 1. Let $(F, X)$ be a soft set over $X$ with the following mapping: $F: X \longrightarrow P(X), F(1)=\{d\}, F(a)=\{a, d\}, F(b)=\{b, d\}, F(c)=\{c, d\}, F(d)=\{d\}$. Then $(F, A)$ is not a soft dual filter. For the subset $C=\{b, c, d\}$ of $A$, the soft set $\left(\left.F\right|_{C}, C\right)$ is a soft dual filter.

Theorem 2. Let $(F, A)$ and $(G, B)$ be two soft $B E$-algebra (soft filter, soft dual filter) over $X$. If $A \cap B \neq \varnothing$, then the intersection $(F ; A) \tilde{\cap}(G ; B)$ is a soft BE-algebra (resp. soft filter, soft dual filter) over $X$.

Proof. Let $(F ; A) \tilde{\cap}(G ; B)=(H, C)$, where $C=A \cap B$ and $H(x)=F(x)$ or $G(x)$ for all $x \in C$. Note that $(H, C)$ is a soft set over $X$ where $H: C \longrightarrow P(X)$. Since $(F, A)$ and $(G, B)$ are soft BE-algebras (resp. soft filter, soft dual filter) over $X$, for all $x \in C, H(x)=F(x)$ or $H(x)=G(x)$ is a subalgebra (resp. soft filter, soft dual filter) over $X$. Therefore $(H, C)=(F, A) \tilde{\cap} G(B)$ is a soft BE-algebra (resp. soft filter, soft dual filter) over $X$.

Theorem 3. Let $(F, A)$ be a soft BE-algebra over $X$. If $(G, B)$ and $(H, C)$ are two soft $F$-filter (soft dual $F$-filter where $X$ is bounded) over $X$ and $B \cap C \neq \varnothing$, then $(G, B) \tilde{\cap}(H, C)$ is soft $F$-filter (soft dual $F$-filter) over $X$.

Proof. It can be done easily similar to proof of the the Theorem 2.

Theorem 4. Let $(F ; A)$ and $(G, B)$ be two soft $B E$-algebra (soft filter, soft dual filter for bounded BE-algebra $X)$ over $X$. If $A \cap B=\varnothing$ then $(F ; A) \tilde{\cup}(G ; B)$ is a soft BE-algebra (resp. soft filter, soft dual filter) over $X$. 
Proof. Let $(F ; A) \tilde{\cup}(G ; B)=(H ; C)$, where $C=A \cup B$ and for all $x \in C$,

$$
H(x)= \begin{cases}F(x), & \text { if } x \in A \backslash B, \\ G(x), & \text { if } x \in B \backslash A, \text { If } x \in A \backslash B \text { or } x \in B \backslash A, \text { then } H(x)=F(x) \text { or } H(x)=G(x) \text { are subalgebra (resp. } \\ F(x) \cup G(x), & \text { if } x \in A \cap B .\end{cases}
$$

soft filter, soft dual filter) over $X$ since $(F ; A)$ and $(G, B)$ soft BE-algebras (soft filters, soft dual filters) over $X$. Since $A \cap B=\varnothing$, there do not exists another case. Hence $(H ; C)=(F ; A) \widetilde{\cup}(G ; B)$ is a soft BE-algebra (resp. soft filter, soft dual filter) over $X$.

If $A \cap B \neq \varnothing$, then generally, Theorem 4 is not valid.

Example 11. Consider the BE-algebra in Example 1.

(1) Let $A=\{1, a\}$ and $F: A \longrightarrow P(X)$ be a mapping such that $F(1)=\{1, d\}, F(a)=\{1, a\}$. Then $(F, A)$ is a soft BEalgebra. Let $B=\{1, b\}$ and $G: B \longrightarrow P(X)$ be a mapping such that $G(1)=\{1, c\}, G(b)=\{1, b\}$. Then $(G, B)$ is a soft BE-algebra. Now, we get $A \cup B=\{1, a, b\}=C, H: C \longrightarrow P(X), H(1)=F(1) \cup G(1)=\{1, c, d\}, H(a)=F(a)=$ $\{1, a\}$ and $H(b)=G(b)=\{1, b\}$. The set $H(1)=\{1, c, d\}$ is not a subalgebra since $c * d=b \notin H(1)$.

(2) Let $A=\{1, a, c\}$ and $F: A \longrightarrow P(X)$ be a mapping such that $F(1)=\{1, b\}, F(a)=\{1, a\}, F(c)=\{1, a, c\}$. Then $(F, A)$ is a soft filter over $X$. Let $B=\{1, b\}$ and $G: B \longrightarrow P(X)$ be a mapping such that $G(1)=\{1, a, c\}, G(b)=\{1, b\}$. Then $(G, B)$ is a soft filter over $X$. We see that $A \cup B=\{1, a, b, c\}=C, H: C \longrightarrow P(X), H(1)=F(1) \cup G(1)=$ $\{1, a, b, c\}$. Also we see that the set $H(1)$ is not a filter of $X$ because $c \in H(1), c * d=b \in H(1)$ but $d \notin H(1)$.

(3) Let $A=\{b, d\}$ and $F: A \longrightarrow P(X)$ be a mapping such that $F(b)=F(d)=\{b, d\}$. Then $(F, A)$ is a soft dual filter over $X$. Let $B=\{c, d\}$ and $G: B \longrightarrow P(X)$ be a mapping such that $G(c)=G(d)=\{c, d\}$. Then $(G, B)$ is a soft dual filter over $X$. We see that $A \cup B=\{b, c, d\}=C, H: C \longrightarrow P(X), H(d)=F(d) \cup G(d)=\{b, c, d\}$. Also we see that the set $H(d)$ is not a soft dual filter over $X$ because $c \in H(d),(c N * 1 N) N=(b * d) * d=b \in H(d)$ but $1 \notin H(d)$.

Theorem 5. Let $(F, A)$ be a soft BE-algebra over $X$. If $(G, B)$ and $(H, C)$ are two soft $F$-filter (soft dual $F$-filter where $X$ is bounded) over $X$ and $B \cap C=\varnothing$, then $(G, B) \tilde{\cup}(H, C)$ is soft $F$-filter (soft dual F-filter) over $X$.

Proof. Similar to the Proof of the Theorem 4.

Theorem 6. Let $(F ; A)$ and $(G, B)$ be two soft BE-algebra (soft filter, soft dual filter for bounded BE-algebra X) over X. Then $(F ; A) \tilde{\wedge}(G ; B)$ is a soft BE-algebra (resp. soft filter, soft dual filter) over $X$.

Proof. We know that $(F ; A) \tilde{\wedge}(G ; B)=(H, A \times B)$ where $H(x, y)=F(x) \cap G(y)$ for all $(x, y) \in A \times B$. For all $x \in A$ and $y \in B$, since $F(x)$ and $G(y)$ are subalgebras (resp.soft filter, soft dual filter for bounded BE-algebra $X), H(x, y)$ is a subalgebra (soft filter, soft dual filter). Therefore $(H, A \times B)=(F ; A) \widetilde{\wedge}(G ; B)$ is a subalgebra (soft filter, soft dual filter).

Example 12. In Example 11 (1), (2) and (3), for the sets $A$ and $B$, Theorem 15 is true.

Theorem 7. Let $(F, A)$ be a soft BE-algebra over X. If $(G, B)$ and $(H, C)$ are two soft $F$-filter (soft dual $F$-filter where $X$ is bounded) over $X$, then $(G, B) \wedge(H, C)$ is soft $F$-filter (soft dual F-filter) over $X$.

Proof. Similar to the Proof of the Theorem 6.

Let $f: X \longrightarrow Y$ be a mapping of BE-algebras. For a soft set $(F, A)$ over $X,(f(F), A)$ is a soft set over $Y$ where $f(F)$ : $A \longrightarrow P(Y)$ is defined by $f(F)(x)=f(F(x))$ for all $x \in A$.

Lemma 1. Let $f: X \longrightarrow Y$ be a BE-algebra homomorphism.

(i) If $A$ is a subalgebra of $X$ then $f(A)$ is a subalgebra of $Y$.

(ii) Let $f$ be one to one and onto. If $A$ is a filter of $X$ then $f(A)$ is a filter of $Y$.

(iii) If $f$ is onto and $X$ be a bounded BE-algebras then $Y$ is a bounded BE-algebra. 
(iv) Let $X$ be a bounded BE-algebra, $f$ be one to one and onto. If $A$ is a dual filter of $X$ then $f(A)$ is a dual filter of $Y$.

Proof. (i) Let $A$ be a subalgebra of $X$ and $u, v$ be any two elements of $f(A)$. There exist $x, y \in A$ such that $f(x)=u$ and $f(y)=v$. Then we have $u * v=f(x) * f(y)=f(x * y) \in f(A)$ since $x * y \in A$.

(ii) Let $A$ be a filter of $X$ and $f$ be one to one and onto. We have $1_{X} \in A$ and $f\left(1_{X}\right)=f\left(1_{X} * 1_{X}\right)=f\left(1_{X}\right) * f\left(1_{X}\right)=1_{Y}$. Hence $1_{Y} \in f(A)$. Now, let $u \in f(A)$ and $u * v \in f(A)$ for $v \in Y$. There exist $x \in A$ and $y \in X$ such that $f(x)=u$ and $f(y)=v$. Then $x * y \in A$ since $u * v=f(x) * f(y)=f(x * y) \in f(A)$. Hence we get $x \in A$ and $x * y \in A$. Since $A$ is a filter of $X$, we get $y \in A$. So $f(y)=v \in f(A)$. That is, $f(A)$ is a filter of $Y$.

(iii) Let $0_{X}$ be the unit of $X$. Then $0_{X} * x=1_{X}$ for all $x \in X$. There exist $y \in X$ such that $f(y)=v$ for all $v \in Y$ since $f$ is onto. In this case, we have $f\left(0_{X} * y\right)=f\left(0_{X}\right) * f(y)$ or $f\left(1_{X}\right)=f\left(0_{X}\right) * v$. Hence we get $1_{Y}=f\left(0_{X}\right) * v$ for all $v \in Y$. So $f\left(0_{X}\right)=0_{Y}$ is the unit of $Y$.

(iv) Since $0_{X} \in A$, we have $f\left(0_{X}\right)=0_{Y} \in f(A)$. Now let $u \in f(A)$ and $(u N * v N) N \in f(A)$ for $v \in Y$. There exist $x \in A$ and $y \in X$ such that $f(x)=u$ and $f(y)=v$. Then since

$$
\begin{aligned}
(u N * v N) N & =\left(\left(u * 0_{Y}\right) *\left(v * 0_{Y}\right)\right) * 0_{Y} \\
& =\left(\left(f(x) * f\left(0_{X}\right)\right) *\left(f(y) * f\left(0_{X}\right)\right)\right) * f\left(0_{X}\right) \\
& =f((x N * y N) N) \in f(A),
\end{aligned}
$$

we see that $(x N * y N) N \in A$. So we get $y \in A$ since $\mathrm{A}$ is a dual filter. Hence $f(y)=v \in f(A)$.

Theorem 8. Let $f: X \longrightarrow Y$ be a BE-algebra homomorphism.

(i) If $(F, A)$ is a soft BE-algebra over $X$, then $(f(F), A)$ is a soft BE-algebra over $Y$.

(ii) Let $f$ be one to one and onto. If $(F, A)$ is a soft filter over $X$ then $(f(F), A)$ is a soft filter over $Y$.

(iii) Let $X$ be a bounded BE-algebra, $f$ be one to one and onto. If $(F, A)$ is a soft dual filter over $X$ then $(f(F), A)$ is a soft dual filter over $Y$.

Proof. It is clear by using the Lemma 1.

Theorem 9. Let $f: X \longrightarrow Y$ be a BE-algebra isomorphism.

(i) Let $(F, A)$ be a soft BE-algebra. If the soft set $(G, B)$ is a soft $F$-filter over $X$ then $(f(G), B)$ is a soft $f(F)$-filter over $Y$.

(ii) Let $X$ be a bounded BE-algebra, $(F, A)$ be a soft BE-algebra over $X$. If $(G, B)$ is a soft dual $F$-filter over $X$ then $(f(G), B)$ is a soft dual $f(F)$-filter over $Y$.

Proof. It is clear by using the Lemma 1.

\section{Competing interests}

The authors declare that they have no competing interests.

\section{Authors' contributions}

All authors have contributed to all parts of the article. All authors read and approved the final manuscript.

\section{Conflict of interest}

The authors declare that there is no conflict of interest regarding the publication of this article. 


\section{References}

[1] S. S. Ahn, N. O. Alshehri, Y. B. Jun, ”Int-soft filters of BE-algebras”, Discrete Dynamics in Nature ans Society, Vol.2013, 1-8.

[2] S. S. Ahn and K. S. So, "On ideals and upper sets in BE-algebras," Scientiae Mathematicae Japonicae, vol. 68, no. 2, pp. 279-285, 2008.

[3] H. Aktaş and N. Çağman, "Soft sets and soft groups," Information Sciences, vol. 177, no. 13, pp. 2726-2735, 2007.

[4] D. Chen, E. C. C. Tsang, D. S. Yeung, and X. Wang, "The parameterization reduction of soft sets and its applications," Computers \& Mathematics with Applications, vol. 49, no. 5-6, pp. 757-763, 2005.

[5] N. Çağman, F. Çitak, and S. Enginoglu, "FP-soft set theory and its applications," Annals of Fuzzy Mathematics and Informatics, vol. 2, no. 2, pp. 219-226, 2011.

[6] Z. Çiloğlu, Y. Çeven, "Commutative and bounded BE-algebras", Algebra, Vol 2013, Article ID 473714, 1-5.

[7] F. Feng, "Soft rough sets applied to multicriteria group decision making," Annals of Fuzzy Mathematics and Informatics, vol. 2, no. 1, pp. 69-80, 2011

[8] Y. Imai and K. Iséki, “On axiom systems of propositional calculi. XIV,” Proceedings of the Japan Academy, vol. 42, pp. 19-22, 1966.

[9] K. Iséki, “An algebra related with a propositional calculus,” Proceedings of the Japan Academy, vol. 42, pp. 26-29, 1966.

[10] Y. B. Jun, ”Soft BCK/BCI-algebras", Computers and Mathematics with Applications 56 (2008), 1408-1413.

[11] Y. B. Jun and S. S. Ahn, “Applications of soft sets in BE-algebras," Algebra, vol. 2013, Article ID 368962, 8 pages, 2013.

[12] Y. B. Jun, C. H. Park, ”Applications of soft sets in ideal theory of BCK/BCI-algebras” Information Sciences 178 (2008), $2466-$ 2475.

[13] Y. B. Jun, S-Z. Song, "Filters of BE-algebras associated with Uni-soft set theory”, Applied Mathematical Sciences, vol.9, 2015, no.84, 4155-4164.

[14] H. S. Kim and Y. H. Kim, “On BE-algebras,” Scientiae Mathematicae Japonicae, vol. 66, no. 1, pp. 113-116, 2007.

[15] P. K. Maji, R. Biswas, and A. R. Roy, "Soft set theory," Computers \& Mathematics with Applications, vol. 45, no. 4-5, pp. 555-562, 2003.

[16] P. K. Maji, A. R. Roy, and R. Biswas, “An application of soft sets in a decision making problem,” Computers \& Mathematics with Applications, vol. 44, no. 8-9, pp. 1077-1083, 2002.

[17] D. Molodtsov, "Soft set theory: first results," Computers \& Mathematics with Applications, vol. 37, no. 4-5, pp. 19-31, 1999.

[18] C. H. Park, Y. B. Jun, M.A.Öztürk, ”Soft WS-algebras”, Commun. Korean Math. Soc. 23, No.3, 313-324, 2008.

[19] L. A. Zadeh, "From circuit theory to system theory," Proceedings of the Institute of Radio Engineers, vol. 50, pp. 856-865, 1962.

[20] L. A. Zadeh, "Fuzzy sets," Information and Computation, vol. 8, pp. 338-353, 1965.

[21] L. A. Zadeh, "Toward a generalized theory of uncertainty (GTU): an outline,” Information Sciences, vol. 172, no. 1-2, pp. 1-40, 2005. 